\title{
Circ-NOLC1 promotes epithelial ovarian cancer tumorigenesis and progression by binding ESRP1 and modulating CDK1 and RhoA expression
}

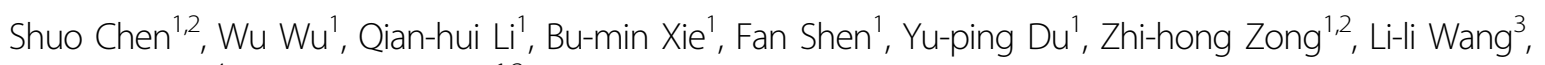
Xiao-qing Wei $^{4}$ and Yang Zhao (1) ${ }^{1,2}$

\begin{abstract}
Circular RNAs (circRNAs) play important roles in cancer tumorigenesis and progression, representing prognostic biomarkers and therapeutic targets. In this case, we demonstrated the role of circ-NOLC1 in epithelial ovarian cancer (EOC). Our results have shown that Circ-NOLC1 expression was higher in EOC tissues than in normal tissues, and was positively associated with FIGO stage, differentiation. Among ovarian cancer cell lines, circ-NOLC1 expression was the highest in A2780, and lowest in CAOV3. Overexpression of circ-NOLC1 in CAOV3 cells increased cell proliferation, migration, and invasion ability, whereas silencing of circ-NOLC1 in A2780 cells had the opposite effect: however, neither circ-NOLC1 downregulation nor overexpression influenced NOLC1 mRNA expression. In nude mice with subcutaneous tumors, circ-NOLC1 downregulation decreased tumor growth. Bioinformatic analysis and RNA-binding protein immunoprecipitation showed that circ-NOLC1 could bind to ESRP1. In addition, the overexpression of circNOLC1 significantly increased ESRP1, RhOA, and CDK1 protein and mRNA expression level; circ-NOLC1 downregulation had the opposite effects. The tumor-promoting effect of circ-NOLC1 was inhibited by knockdown of ESRP1, CDK1, or RhoA expression in circ-NOLC1-overexpressing cells, which might act by modulating RhoA and CDK1 expression. In conclusion, our study demonstrated that Circ-NOLC1 might promote EOC tumorigenesis and development by binding ESRP1 and modulating CDK1 and RhoA expression.
\end{abstract}

\section{Introduction}

Ovarian cancer is a malignant tumor that seriously threatens women's health ${ }^{1-3}$. Asymptomatic clinical appearance in the disease's progression, resulting in widespread disease in the pelvic and abdominal cavity. Therefore, even though surgery, chemotherapy, and biological therapy are used widely to treat ovarian cancer, the 5 -year survival rate remains at only $35-38 \%^{4,5}$. Thus,

\footnotetext{
Correspondence: Yang Zhao (yida.zhaoyang@163.com)

'Department of Gynecologic Oncology Research Office, The Third Affiliated Hospital of Guangzhou Medical University, Guangzhou 510150, China

2Department of Obstetrics and Gynecology, Center for Reproductive Medicine/ Department of Fetal Medicine and Prenatal Diagnosis, Key Laboratory for Major Obstetric Diseases of Guangdong Province, The Third Affiliated Hospital of

Guangzhou Medical University, Guangzhou 510150, China

Full list of author information is available at the end of the article

Edited by Alessandro Rufini
}

research into the mechanism of ovarian cancer development is important for its early detection, diagnosis, and treatment.

The occurrence and development of ovarian cancer is a complex process, comprising multi-step, multi-gene, and multi-factor regulations, especially the regulation of transcription factor regulatory networks, signaling pathways, and regulatory loops. Recent studies have shown that non-coding RNAs also play an important role in the process of ovarian cancer proliferation, apoptosis, invasion, and metastasis ${ }^{6}$.

Non-coding RNAs are RNA molecules in the transcriptome that are not translated into proteins, including small nuclear RNAs, small nucleolar RNAs, microRNAs with relatively small molecular weight (miRNAs), piwiinteracting RNAs, long non-coding RNAs, and circular

\section{(-) The Author(s) 2021}

(c) (i) Open Access This article is licensed under a Creative Commons Attribution 4.0 International License, which permits use, sharing, adaptation, distribution and reproduction cc. in any medium or format, as long as you give appropriate credit to the original author(s) and the source, provide a link to the Creative Commons license, and indicate if changes were made. The images or other third party material in this article are included in the article's Creative Commons license, unless indicated otherwise in a credit line to the material. If material is not included in the article's Creative Commons license and your intended use is not permitted by statutory regulation or exceeds the permitted use, you will need to obtain permission directly from the copyright holder. To view a copy of this license, visit http://creativecommons.org/licenses/by/4.0/. 
RNAs (circRNAs) with relatively large molecular weights. CircRNAs are endogenous RNAs that are widely distributed in eukaryotic cells and have a stable cyclic structure, playing a vital role in the regulation of gene expression. Their unique covalently closed loop structure means that they lack a $5^{\prime}$-end cap structure and $3^{\prime}$ terminal polyadenylation (polyA) tail, which is characterized by tolerance to exonucleases and makes them more stable than homologous linear $\mathrm{RNA}^{7-9}$. CircRNAs play vital roles in almost all kinds of cancer and have the potential to be used as novel biomarkers and therapeutic targets for cancer diagnosis and treatment through their functions as microRNA (miRNA) molecular "sponges"10-12, protein translators ${ }^{13}$, and gene transcription regulators ${ }^{14}$. For example, circTCF25 overexpression promoted cell proliferation and migration by downregulating miR-103a3p/-107 expression and increasing CDK6 (cell-dependent kinase 6) expression in vitro or in vivo ${ }^{11}$. Recent studies also revealed that circRNAs could function as RNAbinding protein sponges ${ }^{15}$. For example, circ-Foxo3 can inhibit CDK2 (cell-dependent kinase 2) and induce cell cycle arrest through a circ-Foxo3-p21-CDK2 ternary complex $^{16}$. However, there are relatively few studies on circRNAs in ovarian cancer.

We detected 12,723 expressed circRNAs in ovarian cancer and normal ovarian tissue through circRNA gene chip, and further screened circRNAs with differential expression up to fivefold, which identified circ-NOLC1. Nucleolar and coiled-body phosphoprotein 1 (NOLC1, also known as Nopp140) is a protein with transcriptionlike activity, which involves in nuclear assembly ${ }^{17}$ and precursor rRNA cleavage ${ }^{18}$, as a novel nucleolar GTPase/ ATPase, it was reported to have a role in the process of the tumorigenesis and progression such as cell cycle, cell proliferation $^{19}$, cell migration, and invasion. Similar to linear RNA, circRNA is also derived from the transcription of parental genes, and its uniqueness lies in the alternative splicing of the non-classical form of RNA precursors. The most common one is exon reverse splicing, that is, circRNA molecules is generated by backsplicing, where downstream exons are spliced to upstream exons in reverse order, leading to a circular transcript ${ }^{20}$. According to gene chip, the precursor RNA of NOLC1 can also form exonic circRNA circ-NOLC1 at exon 1-4 through back-splicing. However, the circular structure of circ-NOLC1, its relationship with NOLC1, and the most important, its role in ovarian cancer tumorigenesis and progression remains unclear.

Therefore, in the present study, we investigated the role of circ-NOLC1 in ovarian cancer and attempted to determine how circ-NOLC1 affects the development of ovarian cancer through a series of in vivo and in vitro experiments.

\section{Results}

The relationship between the circ-NOLC1 expression level and the clinicopathological characteristics of ovarian cancer

Circ-NOLC1 expression in normal ovarian tissues, benign tissues, borderline tumor tissues, and ovarian carcinoma tissues was assessed using quantitative realtime reverse transcription PCR (qRT-PCR). The results showed that circ-NOLC1 expression was significantly higher in borderline and ovarian cancers than in normal and benign ovarian tissues (Fig. $1 \mathrm{~A}, p<0.05$, Table 1 ). Circ-NOLC1 expression in stage II-IV disease was higher than in stage I (Fig. 1B, $p<0.05$ ). In addition, the expression of circ-NOLC1 in the moderately and poor pathological differentiation was higher than that in the well differentiation (Fig. 1C, $" p<0.05$ ). Besides, the expression of circ-NOLC1 was also higher in CA125 positive $\left(>35 \mathrm{U} / \mathrm{ml}\right.$ ) ovarian cancer patients (Fig. $1 \mathrm{D}$, ${ }^{*} p<$ 0.05 , Table 2). These results suggested that circ-NOLC1 involves in the tumorigenesis and progression of ovarian cancer, and may have potential to be treated as diagnostic biomarker.

\section{circ-NOLC1 functions independently from the NOLC1 gene}

We determined circ-NOLC1 expression in six ovarian cancer cell lines using qRT-PCR. Circ-NOLC1 showed the highest expression level in A2780 cells and the lowest in CAOV3 cells (Fig. 2A); therefore, A2780 cells were used for circ-NOLC1 short hairpin RNA (shRNA) transfection, whereas CAOV3 cells were used for circ-NOLC1 overexpression plasmid transfection. QRT-PCR was used to confirm the transfection efficiency (Fig. 2B, C, " $p<0.05$ ). Besides, we found that the NOLC1 mRNA expression level did not change after circ-NOLC1 overexpression (Fig. 2D, $p>0.05$ ) or downregulation (Fig. 2E, $p>0.05$ ), thus we speculated that circ-NOLC1 functions independently of the NOLC1 gene.

\section{The effect of circ-NOLC1 in vitro and in vivo}

Ovarian cancer cell proliferation, migration, and invasion were assessed through CCK8, plate clone, cell apoptosis, wound healing, and transwell assays. The results indicated that circ-NOLC1 overexpression in CAOV3 cells significantly induced cell proliferation (Fig. 3A, $p<0.05$ ) and clonal formation (Fig. 3B, $p<0.05$ ); reduced cell apoptosis (Fig. 3C, $p<0.05$ ); and induced cell migration (Fig. 3D, $p<0.05$ ) and invasion (Fig. 3E, $p<$ $0.05)$, which means that circ-NOLC1 functions as an "oncogene" in ovarian cancer. Compared with the negative control, after circ-NOLC1 downregulation using circNOLC1 shRNA transfection in A2780 cells, cell proliferation (Fig. 4A, $p<0.05$ ) and clonal formation (Fig. 4B, $p<0.05)$ were significantly inhibited, whereas cell 
A
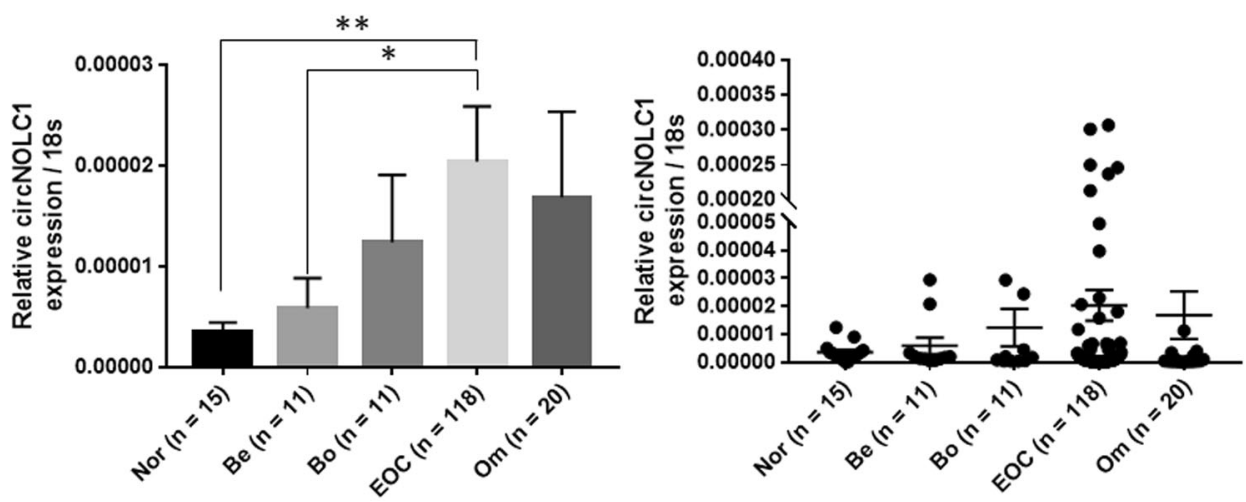

B
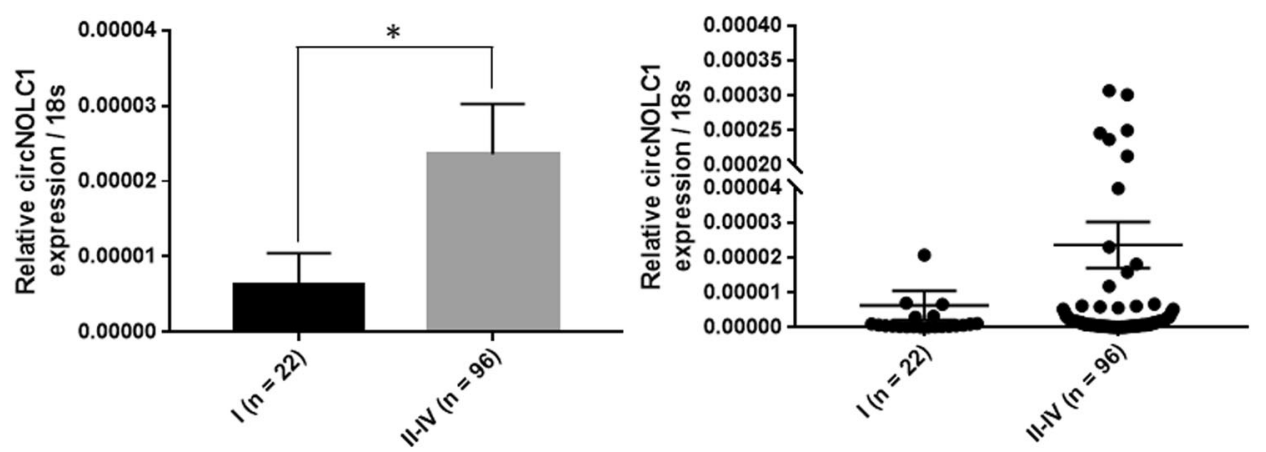

C

FIGO Stage

FIGO Stage
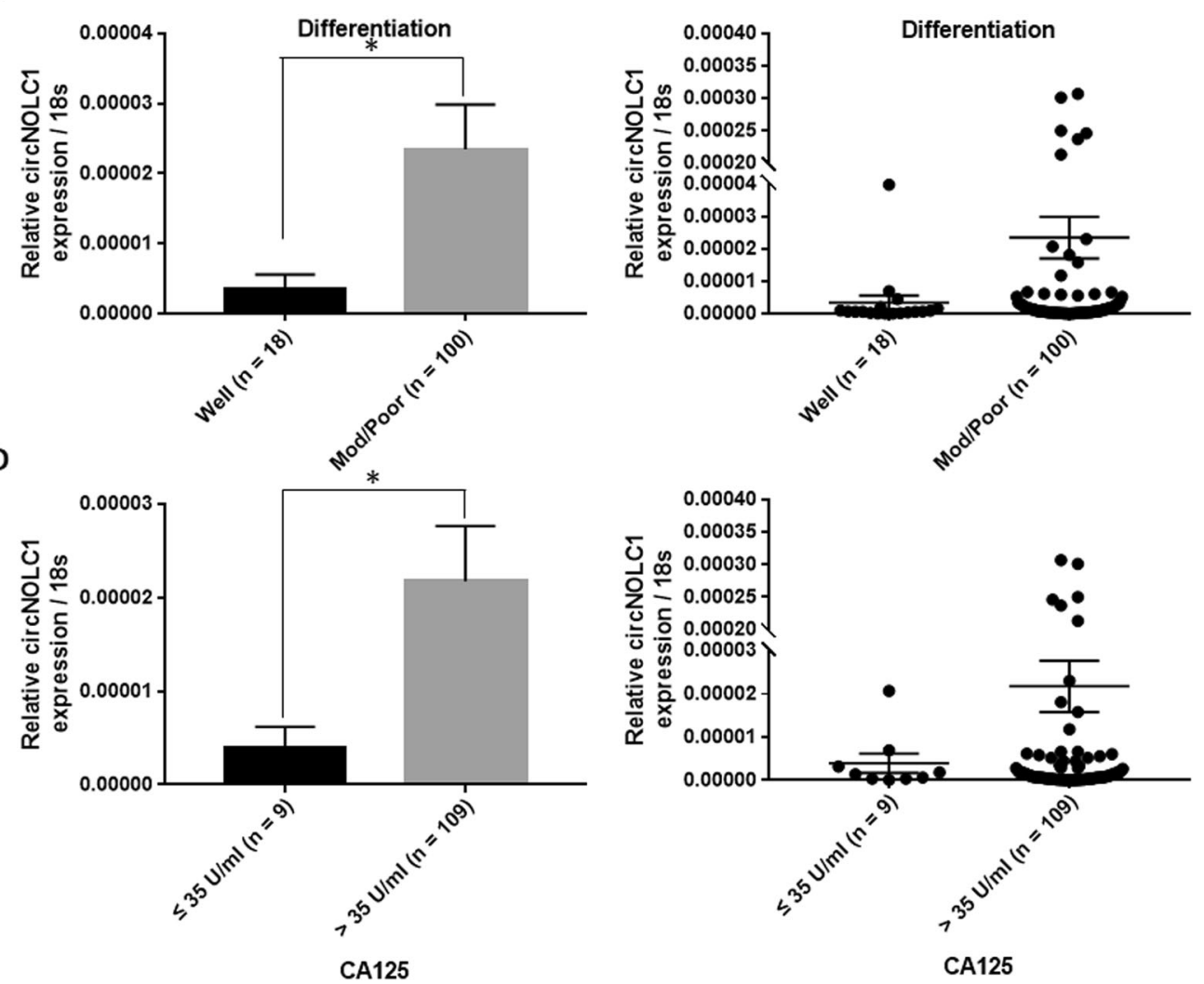

Fig. 1 (See legend on next page.) 
(see figure on previous page)

Fig. 1 The relationship between circ-NOLC1 expression and the clinicopathological characteristics of ovarian cancer. A Circ-NOLC1

expression in normal ovaries $(n=15)$, benign ovarian tumors $(n=11)$, borderline tumors $(n=11)$ and ovarian carcinomas $(n=118)$ was analyzed by qRT-PCR. B FIGO stages: The expression of circ-NOLC1 in stage II-IV $(n=96)$ was higher than that in stage I $(n=22)$. C Pathology classification: circNOLC1 expression in the moderate (Mod) and Poor differentiation $(n=100)$ was higher than in the well differentiation $(n=18)$. D CA125: circ-NOLC1 expression in the CA125 positive $(n=109)$ was higher than in the CA125 negative $(n=9)$ patients. Data are expressed as the mean \pm SD. Correlation between circ-NOLC1 expression and clinicopathological characteristics were examined using the $x^{2}$ test. ${ }^{*} p<0.05$.

Table 1 circ-NOLC1 expression in different ovarian tissues.

\begin{tabular}{llll}
\hline Groups & $\boldsymbol{N}$ & TDRG1 expression/18 s & $\boldsymbol{P}$ value \\
\hline Normal ovary & 15 & $3.57013 \mathrm{E}-06 \pm 3.29678 \mathrm{E}-06$ & $\mathbf{0 . 0 0 1 5}$ \\
Benign ovarian tumor & 11 & $5.86156 \mathrm{E}-06 \pm 9.77477 \mathrm{E}-06$ & $\mathbf{0 . 0 1 0 8}$ \\
Borderline ovarian tumor & 11 & $1.23827 \mathrm{E}-05 \pm 2.21106 \mathrm{E}-05$ & \\
Ovarian carcinoma & 118 & $2.03776 \mathrm{E}-05 \pm 5.94288 \mathrm{E}-05$ & \\
Omentum tumor & 20 & $1.68522 \mathrm{E}-05 \pm 3.81715 \mathrm{E}-05$ & \\
\hline
\end{tabular}

Bold and italics means $P<0.05$

apoptosis was induced (Fig. 4C, $p<0.05$ ), and cell migration (Fig. 4D, E, $p<0.05$, Supplementary videos $1 \&$ 2 ) and invasion (Fig. 4F, $p<0.05$ ) were decreased. Nude mice xenograft assays demonstrated that the tumorigenicity was inhibited in mice injected with circ-NOLC1downregulated A2780 cells (Fig. 5D; $p<0.05$ ) compared with that in the control group (Fig. 5A, B), and the tumor volumes were smaller in the circ-NOLC1 downregulation group (Fig. 5 C; $p<0.05$ ).

\section{The correlation between circ-NOLC1 and ESRP1}

Bioinformatic prediction showed that circ-NOLC1 has binding sites for miR-326-5p, miR-330, miR-370, and miR-9-5p (Circular RNA Interactome, https:// circinteractome.irp.nia.nih.gov/), and these miRNAs also have binding sites for RhoA and CDK1 mRNA (mirDIP, http://ophid.utoronto.ca/mirDIP/index.jsp). Using western blotting, we confirmed that circ-NOLC1 overexpression induced RhoA and CDK1 protein expression, while circ-NOLC1 downregulation inhibited RhoA and CDK1 protein expression both in vivo and in vitro (Figs. 5E and 6A). However, the results of dual-luciferase reporter assay indicated that circ-NOLC1 might not bind with miR-326-5p, miR-330, miR-370, and miR-9-5p (Supplementary Fig. 1). Bioinformatic prediction software (catRAPID, http://s.tartaglialab.com/page/ catrapid_group) revealed that ESRP1 could bind with circ-NOLC1 (Supplementary Fig. 2), which we confirmed experimentally using a RIP assay. RNA obtained from the RIP assay using an anti-ESRP1 antibody was subjected to qPCR analysis, which demonstrated the enrichment of circ-NOLC1 (Fig. 6B). We also confirmed that circ-
NOLC1 overexpression could induce ESRP1 protein expression, whereas circ-NOLC1 downregulation inhibited ESRP1 protein expression. Besides, through qRTPCR, we confirmed that circ-NOLC1 overexpression induced ESRP1, RhoA, and CDK1 mRNA expression, whereas circ-NOLC1 downregulation inhibited ESRP1, RhoA, and CDK1 mRNA expression (Fig. 6C, D).

\section{Silencing ESRP1, CDK1 or RhoA could reverse circ-NOLC1's function as an oncogene}

Silencing ESRP1 in circ-NOLC1-overexpressing CAOV3 cells significantly reduced cell viability (Fig. 7A, $p<0.05$ ) and clonal formation (Fig. 7B, $p<0.05$ ), induced apoptosis (Fig. 7C, $p<0.05$ ), and reduced cell migration (Fig. 7D, $p$ $<0.05$ ) and invasion (Fig. 7E, $p<0.05$ ). Furthermore, knockdown of ESRP1 reversed the ability of circ-NOLC1 to upregulate RhoA and CDK1 protein expression in circNOLC1-overexpressing CAOV3 cells (Fig. 7F). These results showed that ESRP1 has a key role during the tumor-promoting process of circ-NOLC1. What's more, we also found significantly reduced cell viability (Fig. 8A, $p<0.05$ ), cell migration (Fig. $8 \mathrm{~B}, p<0.05$ ) and invasion (Fig. $8 \mathrm{C}, p<0.05$ ) ability neither after $C D K 1$ or RhoA downregulation in circ-NOLC1-overexpressing CAOV3 cells, however, ESRP1 protein expression level did not change after neither after $C D K 1$ or RhoA knockdown (Fig. 8D). Thus, we hypothesized that circ-NOLC1 might participate in ovarian cancer tumorigenesis and progression by binding the ESRP1, thus modulating RhoA and CDK1 expression.

\section{Discussion}

The results of the present study have showed that circNOLC1 expression was significantly higher in ovarian cancer tissues than in normal ovarian tissues. CircNOLC1 expression in FIGO stage II-IV disease was higher than that in stage I, and in moderate and poor differentiation than in the well differentiation ovarian cancer tissues. Besides, the expression of $\operatorname{circ-NOLC1}$ was also higher in CA125 positive ovarian cancer patients. Thus, we hypothesized that circ-NOLC1 might participate in ovarian cancer tumorigenesis and progression.

Furthermore, in vitro, circ-NOLC1 overexpression induced cell growth, migration, and invasion, whereas silencing of circ-NOLC1 decreased cell growth, migration, 
Table 2 Correlation of circ-NOLC1 expression with different clinicopathological features of ovarian carcinoma.

\begin{tabular}{|c|c|c|c|}
\hline Clinicopathological features & $N$ & circ-NOLC1 expression/18 s & $P$ value \\
\hline The pathology types & & & 0.2795 \\
\hline Serous carcinoma & 86 & $2.20741 \mathrm{E}-05 \pm 6.38584 \mathrm{E}-05$ & \\
\hline The other pathology types & 32 & $1.58183 \mathrm{E}-05 \pm 4.60309 \mathrm{E}-05$ & \\
\hline Age & & & 0.2531 \\
\hline$\leq 52$ & 62 & $2.38226 \mathrm{E}-05 \pm 6.41964 \mathrm{E}-05$ & \\
\hline$>52$ & 56 & $1.65634 \mathrm{E}-05 \pm 5.39825 \mathrm{E}-05$ & \\
\hline CA125 & & & 0.0029 \\
\hline$\leq 35 \mathrm{U} / \mathrm{ml}$ & 9 & $3.97132 \mathrm{E}-06 \pm 6.6421 \mathrm{E}-06$ & \\
\hline$>35 \mathrm{U} / \mathrm{ml}$ & 109 & $2.17322 \mathrm{E}-05 \pm 6.16323 \mathrm{E}-05$ & \\
\hline FIGO stages & & & 0.0144 \\
\hline । & 22 & $6.29372 \mathrm{E}-06 \pm 1.94561 \mathrm{E}-05$ & \\
\hline II-IV & 96 & $2.36051 \mathrm{E}-05 \pm 6.48809 \mathrm{E}-05$ & \\
\hline Pathology classification & & & 0.0018 \\
\hline Well & 18 & $3.39141 \mathrm{E}-06 \pm 9.26029 \mathrm{E}-06$ & \\
\hline Mod + poor & 100 & $2.34351 \mathrm{E}-05 \pm 6.40101 \mathrm{E}-05$ & \\
\hline
\end{tabular}

Bold and italics means $P<0.05$.

and invasion. In addition, nude mice xenograft assays also demonstrated reduced tumorigenicity in mice injected with circ-NOLC1-downregulated A2780 cells. However, neither circ-NOLC1 downregulation nor overexpression influence NOLC1 mRNA expression. Thus, we hypothesized that circ-NOLC1 functions independently of NOLC1, which prompted the question: how does circNOLC1 function as an oncogene?

CircRNAs have attracted much attention recently, and studies have shown that circRNAs participate in cancer development in many ways. The most common function of circRNAs is as miRNA sponges, thereby releasing the inhibition of the miRNAs' target mRNAs. Through bioinformatic analysis, we found that circ-NOLC1 has binding sites for a series of miRNAs such as miR-326-5p, miR-330, miR-370, and miR-9-5p, and that these miRNA also have binding sites with RhoA and CDK1.

Ras homologous family member A (RhoA) is a small molecule G protein/GTPase $(\sim 22 \mathrm{kDa})^{21}$ that regulates the cytoskeleton and affects cell migration by activating ROCK and other effector proteins ${ }^{22}$. Studies have shown that this mode of regulation is involved in the invasion and metastasis of various tumors, such as gastric cancer ${ }^{23}$ and breast cancer ${ }^{24}$. Our previous research found that RhoA is overexpressed in ovarian epithelial cancer, and its expression is positively associated with FIGO stage and differentiation. RhoA not only affects ovarian cancer invasion and metastasis, but also affects the occurrence of ovarian cancer by regulating apoptosis-related genes ${ }^{25}$. Cyclin-dependent kinase 1 (CDK1 or CDC2), a member of the serine/threonine protein kinase family, is the checkpoint protein for the cell cycle from G2 to M phase and is involved in a variety of tumorigenic developments $^{26}$. Our previous research also proved that CDK1 participates in ovarian cancer tumorigenesis and progression $^{27}$. We first assessed RhoA and CDK1 expression levels after circ-NOLC1 overexpression and downregulation. After circ-NOLC1 overexpression or downregulation, RhoA and CDK1 protein levels were significantly upregulated or inhibited, respectively. Thus, we hypothesized that $\operatorname{circ-NOLC1}$ could promote ovarian cancer development by binding with miRNAs that inhibit the translation of CDK1 and RhoA. However, dualluciferase reporter assays demonstrated that circ-NOLC1 might not directly bind with miR-326-5p, miR-330, miR370 , or miR-9-5p, leaving open the question of how circNOLC1 influences RhoA and CDK1 expression.

Studies have shown that despite functioning as miRNA sponges, circRNAs might also function in other ways, such as regulating transcription and acting as enzyme scaffolds. Recently, researchers showed that circRNAs could function by enabling circRNA-protein interactions $^{28}$. For example, Abdelmohsen et al. ${ }^{29}$ reported that the extensive binding of $\mathrm{Hu}$-Antigen $\mathrm{R}$ ( $\mathrm{HuR}$ ) to circ$P A B P N 1$ prevents its binding to poly(A) binding protein nuclear 1 (PABPN1) mRNA and reduces PABPN1 translation. In addition, our previous research reported that circRhoC functions not only as a miR-302e sponge to positively regulate VEGFA protein expression, but may also directly bind and modulate VEGFA expression to 


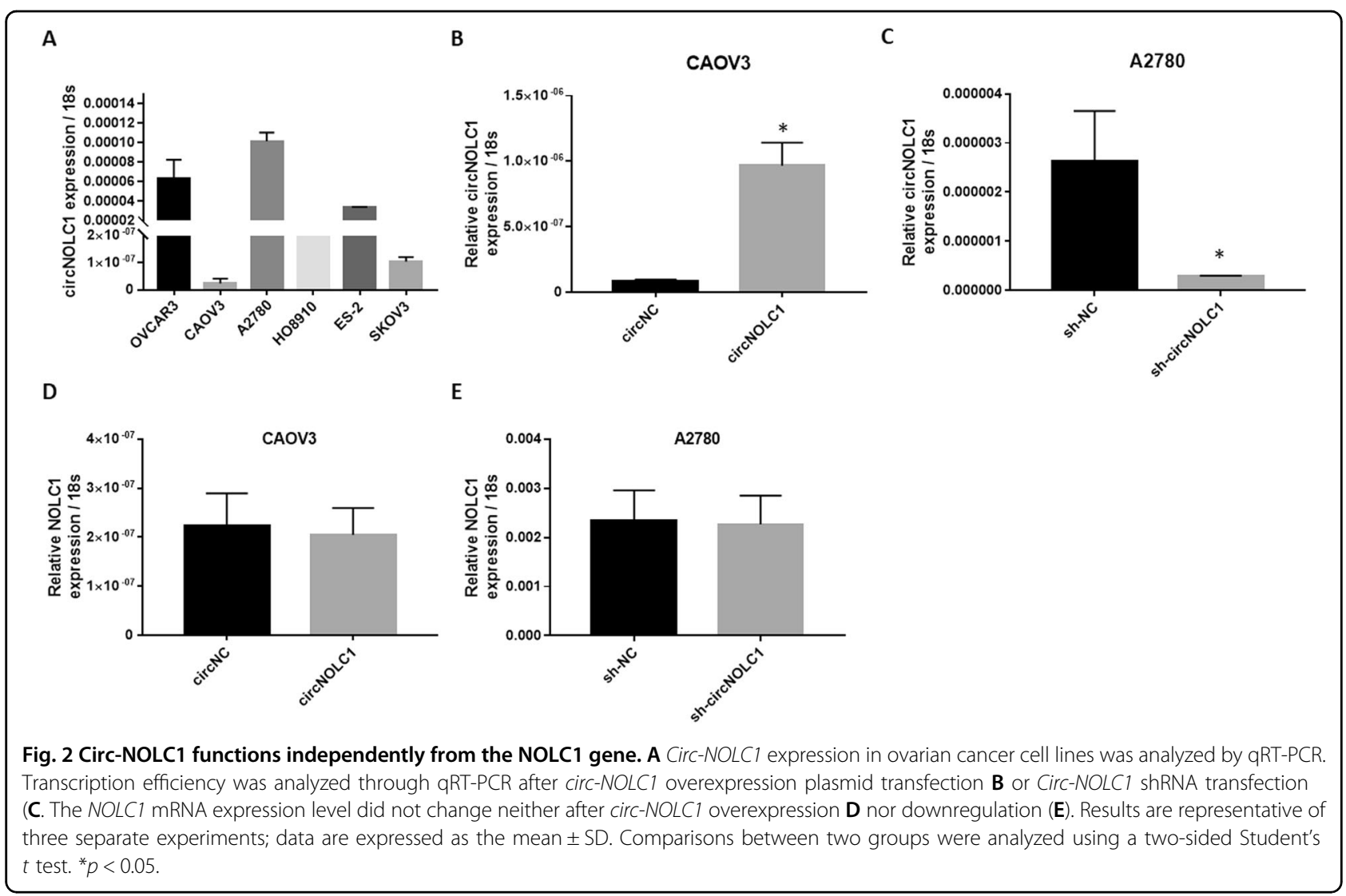

promote ovarian cancer development ${ }^{30}$. Recently, Sun et al. ${ }^{31}$ reported that circMYBL2 regulates the translation efficiency of oncogene FLT3 mRNA by recruiting RNAbinding protein PTBP1, thereby promoting the occurrence and development of FLT3-ITD mutant leukemia, which demonstrated for the first time that circRNA could play a positive regulatory role in the translation process in the form of an RNA-protein complex. Using bioinformatic analysis, we found that circ-NOLC1 could also bind with proteins such as ESRP1. An extensive literature search revealed that ESRP1 was also overexpressed in ovarian cancer tissues; therefore, we suggested that circNOLC1 could bind with ESRP1 and modulating CDK1 and RhoA expression. Through RIP assay we confirmed that $\operatorname{circ-NOLC1}$ binds with ESRP1. In support of this, we found that circ-NOLC1 overexpression increased the ESRP1 protein level, whereas circ-NOLC1 downregulation has the opposite effect. In addition, the tumor-promoting effect of circ-NOLC1 was reduced after ESRP1, CDK1, or RhoA knockdown in circ-NOLC1-overexpressing cells, thus we hypothesized that circ-NOLC1 might function as an oncogene through binding and modulating ESRP1, CDK1, and RhoA levels.

ESRP1 is an epithelial cell-specific RNA-binding protein that regulates alternative splicing of multiple genes. Studies indicate that ESRP1 has a critical role in tumor motility and invasiveness by facilitating cancer cell escape from primary tumors ${ }^{32}$. Studies also showed that ESRP1 promotes breast cancer metastasis by regulating CD44 mRNA alternative splicing. Besides, high ESRP1 expression indicates a shorter overall survival ${ }^{33}$. Fagoonee et al. ${ }^{34}$ reported that the overexpression of ESRP1 promotes colorectal cancer development by stimulating cell proliferation. Jeong et al. ${ }^{35}$ reported that ESRP1 is overexpressed in ovarian cancer and participates in the epithelial-mesenchymal transition process. In addition, after inhibiting ESRP1 expression in circNOLC1 overexpressing CAOV3 cells, RhoA and CDK1 protein levels were decreased, while CDK1 or RhoA downregulation has no effect on ESRP1 protein level, which confirmed our suggestion that circ-NOLC1 functions as an oncogene by binding ESRP1 and modulating CDK1 and RhoA levels. Circ-NOLC1 may bind to ESRP1 protein, and inhibit protein degradation by affecting its protein stabilization in the form of an RNAprotein complex, thereby promoting the expression of downstream CDK1 and RhoA.

\section{Conclusions}

We demonstrated that circ-NOLC1 promotes ovarian cancer tumorigenesis and development by binding to ESRP1 and modulating CDK1 and RhoA levels. This is the 


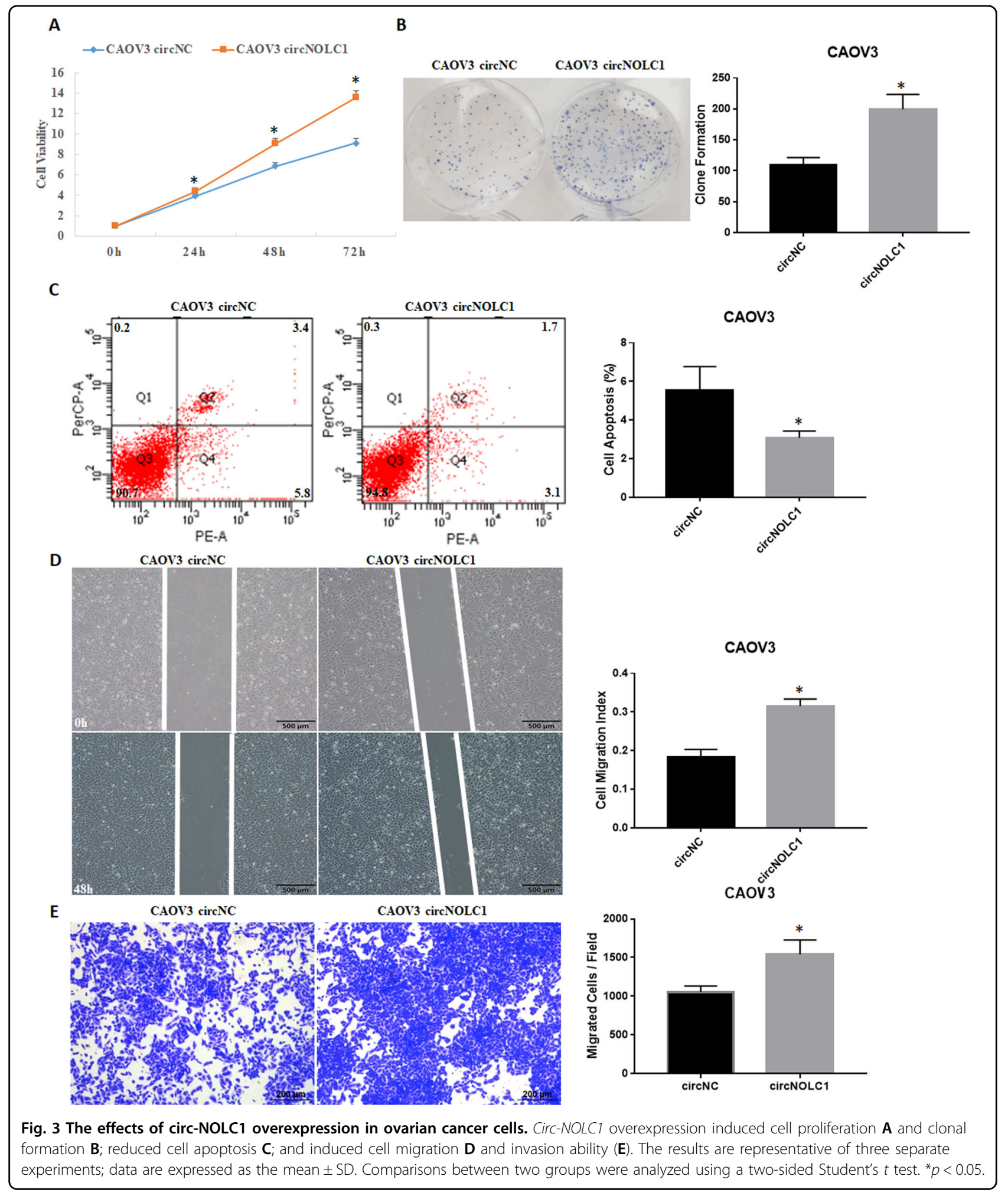

first study to suggest that circ-NOLC1 functions in ovarian cancer and to propose its potential molecular mechanism. Thus, our findings provide a new perspective for the early diagnosis and targeted therapy of ovarian cancer.

\section{Materials and methods}

Ovarian cancer specimens

Normal ovaries, benign ovarian tumors, borderline tumors, and ovarian cancer tissues were collected from 


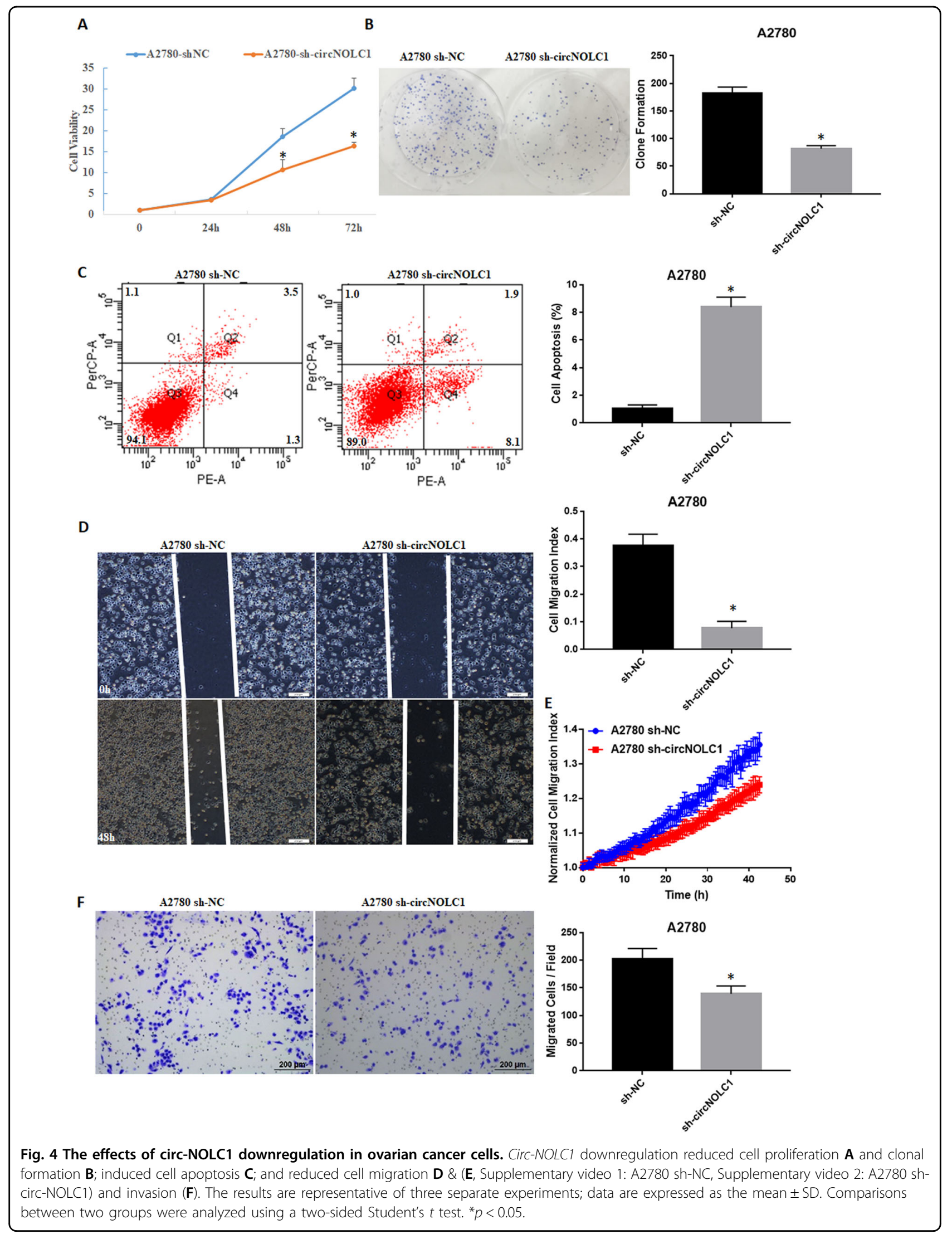



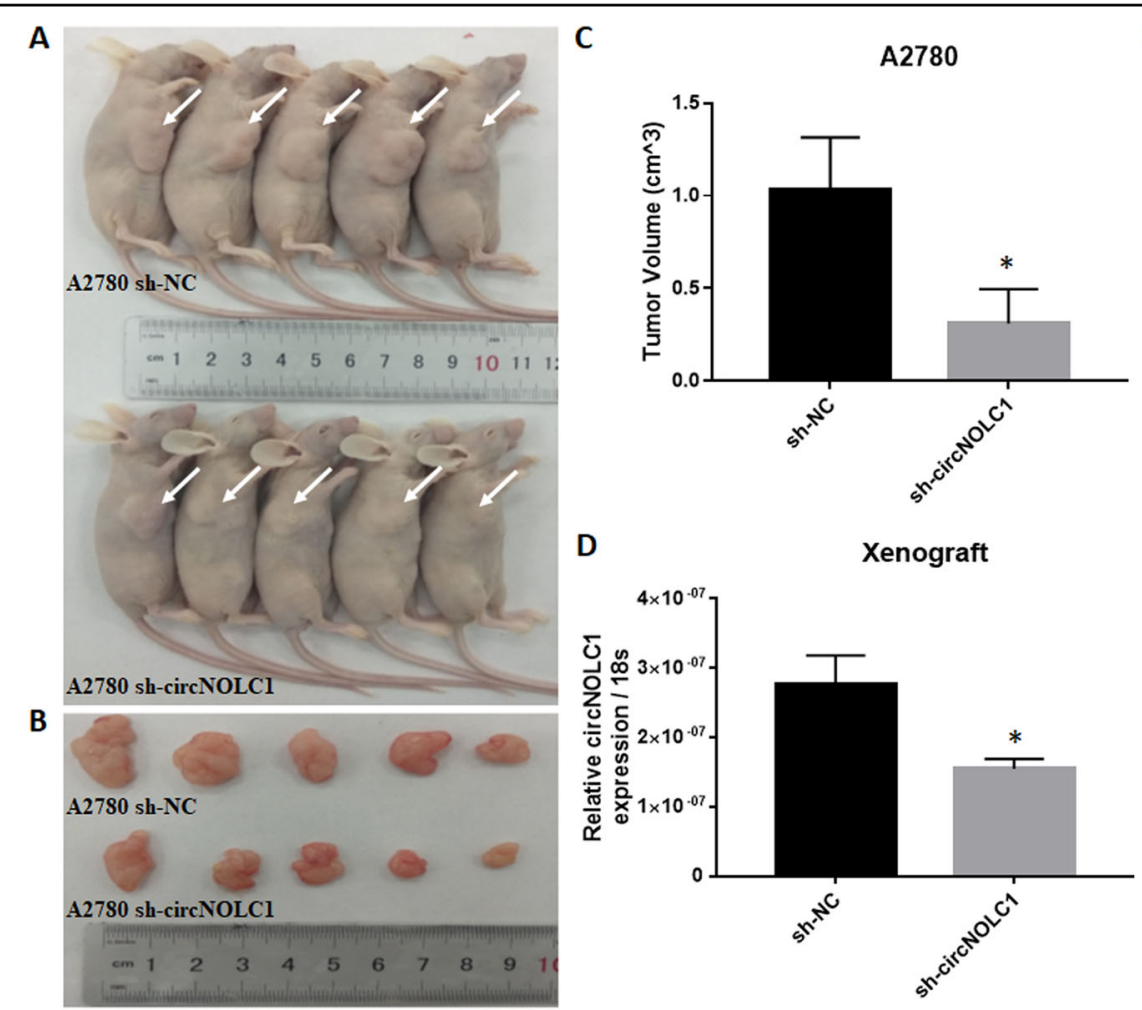

E

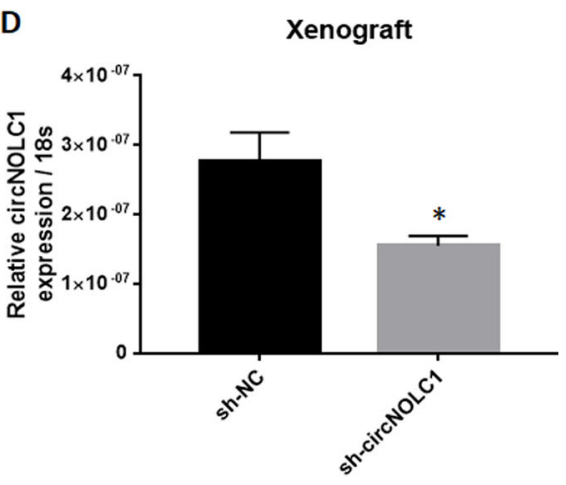

Fig. 5 The effect of circ-NOLC1 downregulation in vivo. Circ-NOLC1 downregulation reduced tumorigenicity in nude mice (A \& B). The tumor volumes were smaller in the sh-circ-NOLC1 group than in sh-NC group (C), Circ-NOLC1 expression (D) and ESRP1, RhoA, CDK1 protein level were all downregulated in sh-circ-NOLC1 group than in sh-NC group (E). Comparisons between two groups were analyzed using a two-sided Student's $t$ test. ${ }^{*} p<0.05$.

patients who accept gynecological surgery at the First Affiliated Hospital of China Medical University (Shenyang, China). After collection, samples were frozen in liquid nitrogen immediately. The tissue specimen was confirmed by two pathologists independently. The study was approved by the Ethics Committee of the China Medical University (no: 2018-132).

\section{Cell culture and transfection}

Human ovarian cancer cell lines A2780, CAOV3, ES-2, HO8910, OVCAR3, and SKOV3 were purchased from Jennio Biotech (Guangzhou, China) or ATCC (Manassas, VA, USA). A2780 and ES-2 cells were cultured in Dulbecco's modified Eagle's medium (HyClone, Logan, UT, USA); SKOV3 cells were cultured in McCoys' $5 \mathrm{~A}$; whereas CAOV3, OVCAR3, and HO8910 cells were cultured in Roswell Park Memorial Institute-1640 (HyClone). All media were supplemented with $10 \%$ fetal bovine serum (FBS) and penicillin/streptomycin (100 U/ $\mathrm{mL}$ ). The cells were incubated with $5 \% \mathrm{CO}_{2}$ at $37^{\circ} \mathrm{C}$. Lipofectamine 3000 was used for plasmid or small interfering RNA transfection according to the manufacturer's instructions (Invitrogen, Carlsbad, CA, USA). CircNOLC1 was upregulated using a circ-NOLC1 overexpressing plasmid, and was knocked down using a circ-NOLC1 shRNA expressing plasmid (the sequence is shown in the Supplemental file). Puromycin was used to select stable clonal cell lines. The sequence of si-ESRP1 was $5^{\prime}$-CUGAAGAAGUGGUGGCCUUdTdT-3', and $3^{\prime}$ AAGGCCACCACUUCUUCAGdTdT-5'.

\section{CCK8 (Cell Counting Kit-8) assay}

Cell viability was examined using the CCK8 assay. Cells were resuspended and seeded in 96-well plates at a density of 1500 cells per well. After the cells were plated, $10 \mu \mathrm{L}$ of CCK8 solution (Bintech Co., Ltd., Shanghai, China) was added into each well at $0,24,48$, and $72 \mathrm{~h}$, and then incubated in $5 \% \mathrm{CO}_{2}$ and $37^{\circ} \mathrm{C}$ for $2 \mathrm{~h}$. The absorbance at $450 \mathrm{~nm}$ was measured using a microplate spectrophotometer (BioTek Instruments, Winooski, VT, USA).

\section{Wound-healing assay}

Cell migration was detected using a wound-healing assay. The cells were resuspended and seeded in six-well plates at a density of $5 \times 10^{5}$ cells per well. When the cells reached $80 \%$ confluence, each well was scored vertically using a 200 $\mu \mathrm{L}$ pipette tip. The excess suspended cells were then rinsed off using phosphate-buffered saline (PBS) three times. Two 


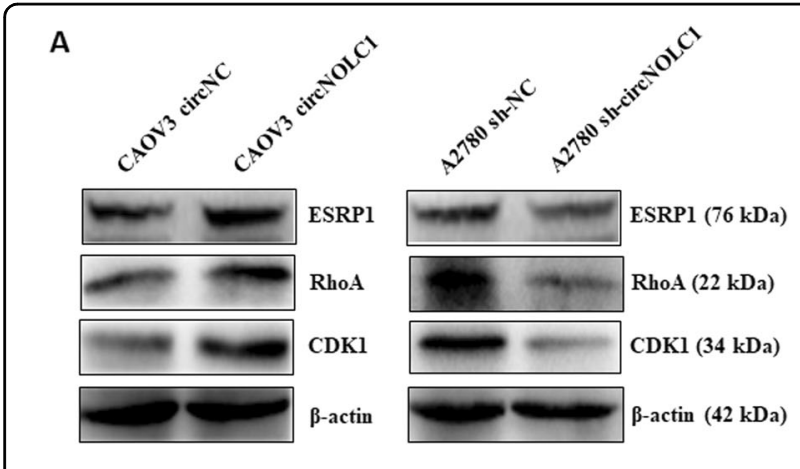

C

CAOV 3

B
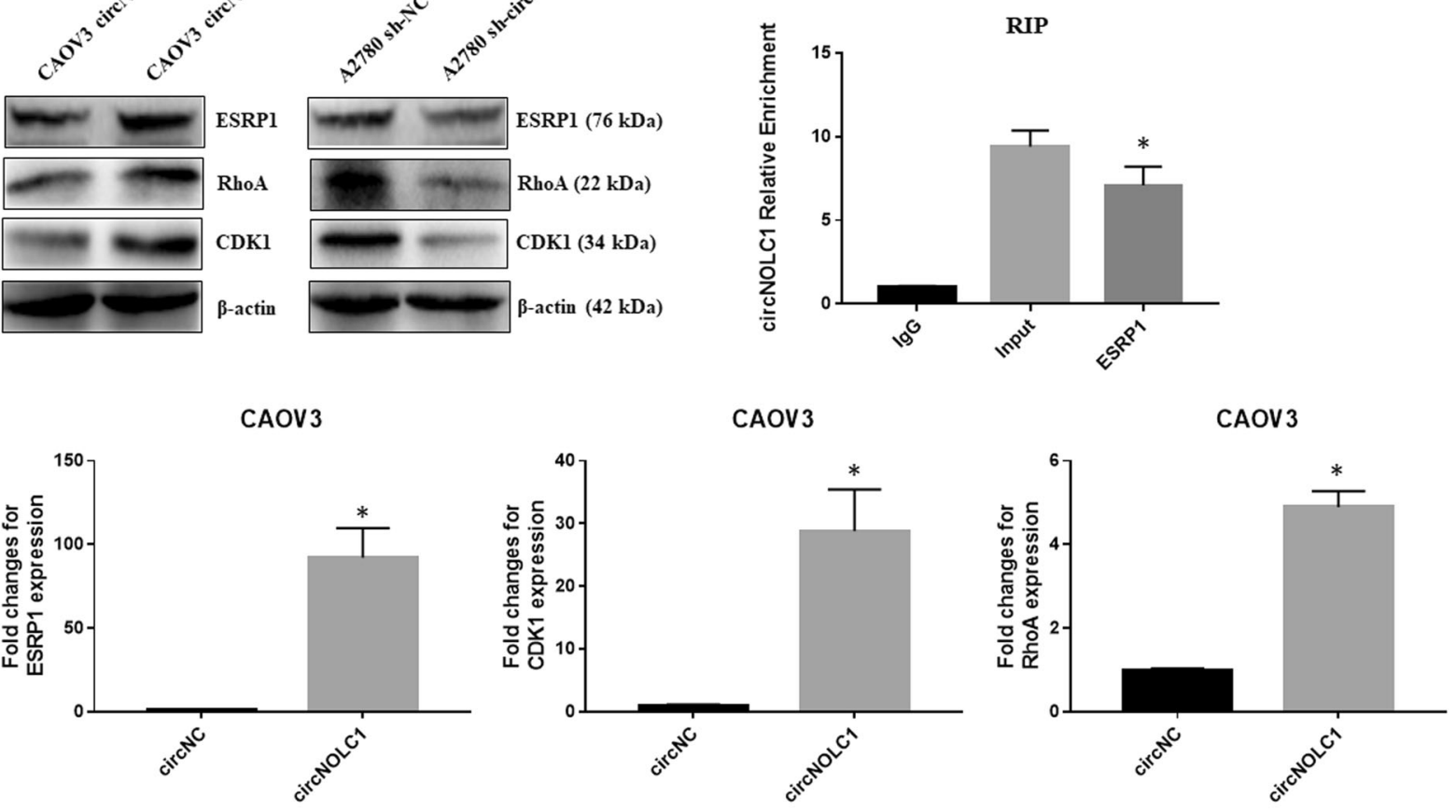

D

A2780

A2780

A2780
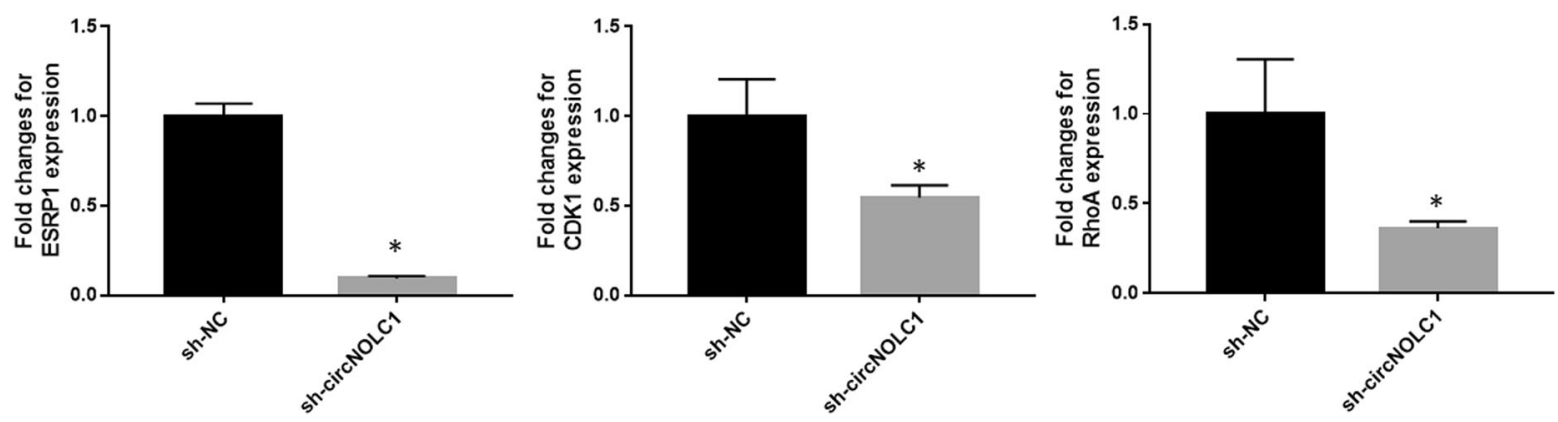

Fig. 6 The correlation between circ-NOLC1 and ESRP1. Circ-NOLC1 overexpression or downregulation induced or inhibited ESRP1, RhOA and CDK1 protein $\mathbf{A}$ or mRNA expression $\mathbf{C}-\mathbf{D}$, respectively. RIP assay confirmed the enrichment of circ-NOLC1 (B). Comparisons between two groups were analyzed using a two-sided Student's $t$ test. ${ }^{*} p<0.05$.

milliliters of the serum-free medium was then added to each well and the cells were incubated at $5 \% \mathrm{CO}_{2}$ and $37^{\circ} \mathrm{C}$. The wounded cells were photographed under an optical microscope at 0,24 , and $48 \mathrm{~h}$, and the wound area was measured using Image $\mathrm{J}$ software (National Institutes of Health, Bethesda, MD, USA). The wound-healing rate was calculated as follows: (original wound area-wound area at each time point)/original wound area $\times 100 \%$.

\section{Cell migration assay by using cytation 5 cell imaging multi- mode reader}

Cells were plated $(40,000$ cells/well) in white, 96-well clear-bottom plates (Corning) with $100 \mu \mathrm{L}$ final volume of complete media. When the cells were plated,
AutoScratch $^{\mathrm{TM}}$ (BioTek) was used to form scratch, the excess suspended cells were then rinsed off using PBS three times, after which, $100 \mu \mathrm{L}$ serum-free medium was then added to each well and the 96-well plate was incubated and imaged (every $30 \mathrm{~min}$ ) at $5 \% \mathrm{CO}_{2}$ and $37^{\circ} \mathrm{C}$ using a Cytation 5 Cell Imaging Multi-Mode Reader (BioTek Instruments, Winooski, VT, USA).

\section{Invasion assay}

The cell invasion ability was measured using a Transwell chamber. Matrigel matrix (BD Biosciences, San Jose, CA, USA) was diluted 1:10 with serum-free medium. The prepared diluted Matrigel was then uniformly added to the upper chamber, and then the Transwell chamber was 

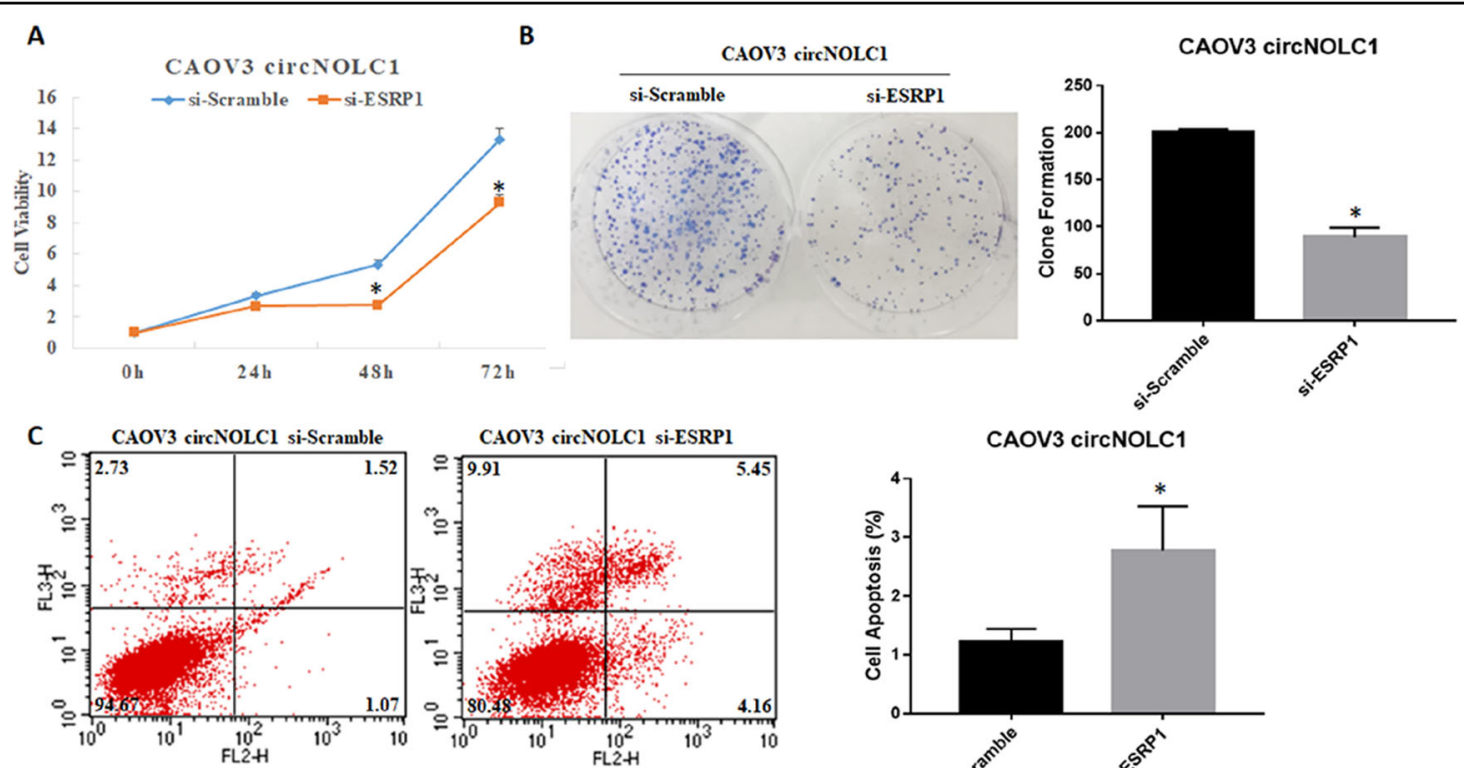

D CAOV3 circNOLCl + si-Scramble

CAOV3 circNOLCl + si-ESRP1
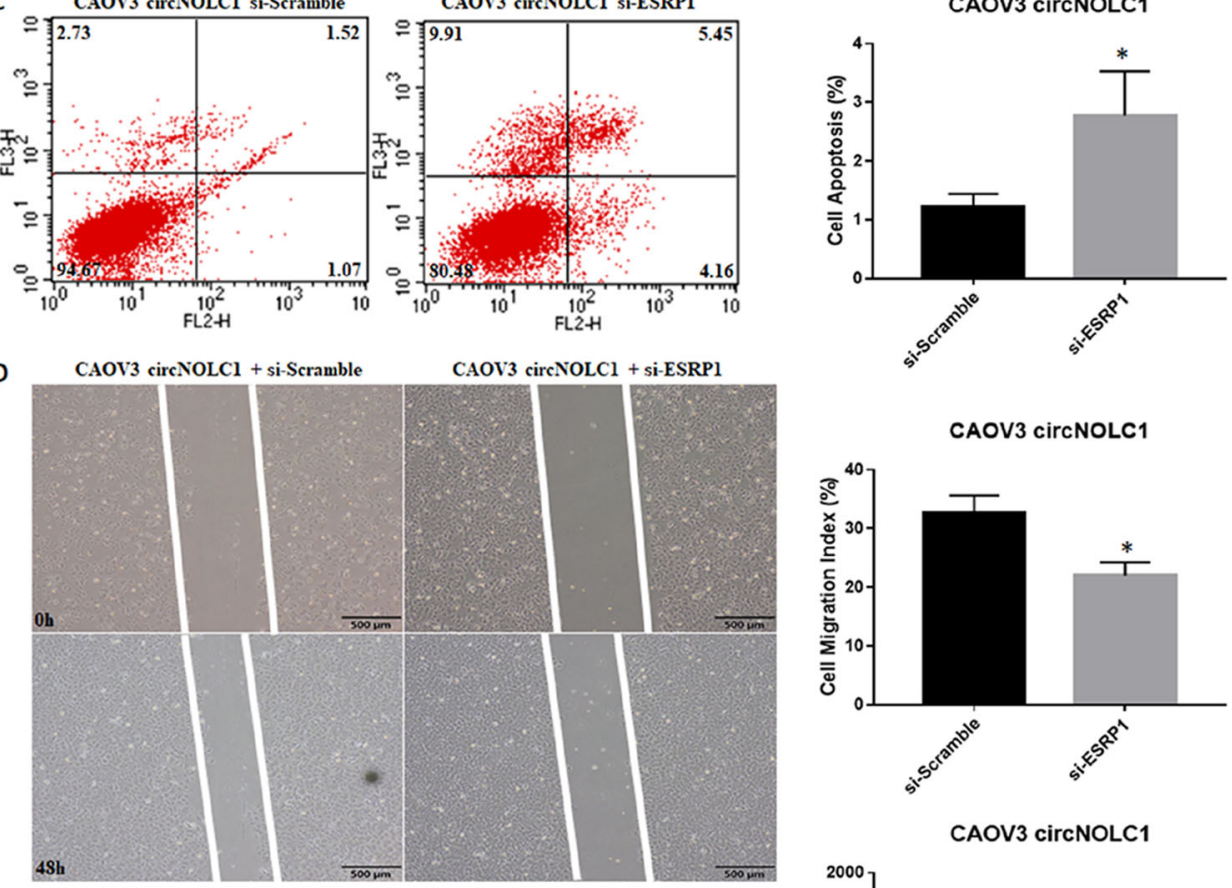

E
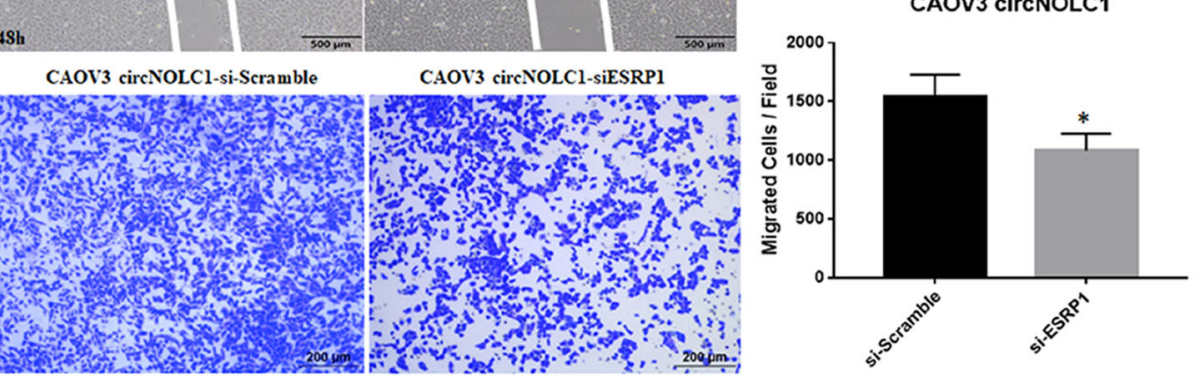

$\mathbf{F}$

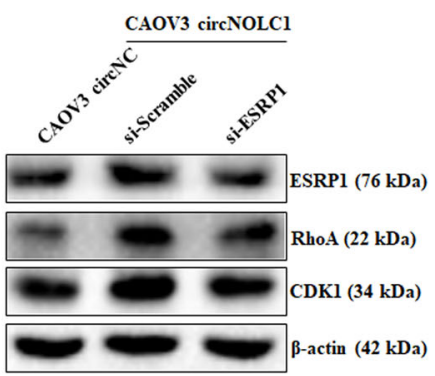

Fig. 7 Silencing ESRP1 reversed circ-NOLC1's function as an oncogene. Silencing ESRP1 inhibited cell viability $\mathbf{A}$ and clonal formation $\mathbf{B}$, induced apoptosis $\mathbf{C}$, and reduced cell migration $\mathbf{D}$ and invasion $\mathbf{E}$ in circ-NOLC1-overexpressing CAOV3 cells, and inhibited RhoA and CDK1 protein expression $(\mathbf{F})$. Results are representative of three separate experiments; data are expressed as the mean $\pm \mathrm{SD}$. Comparisons between two groups were analyzed using a two-sided Student's $t$ test. ${ }^{*} p<0.05$. 


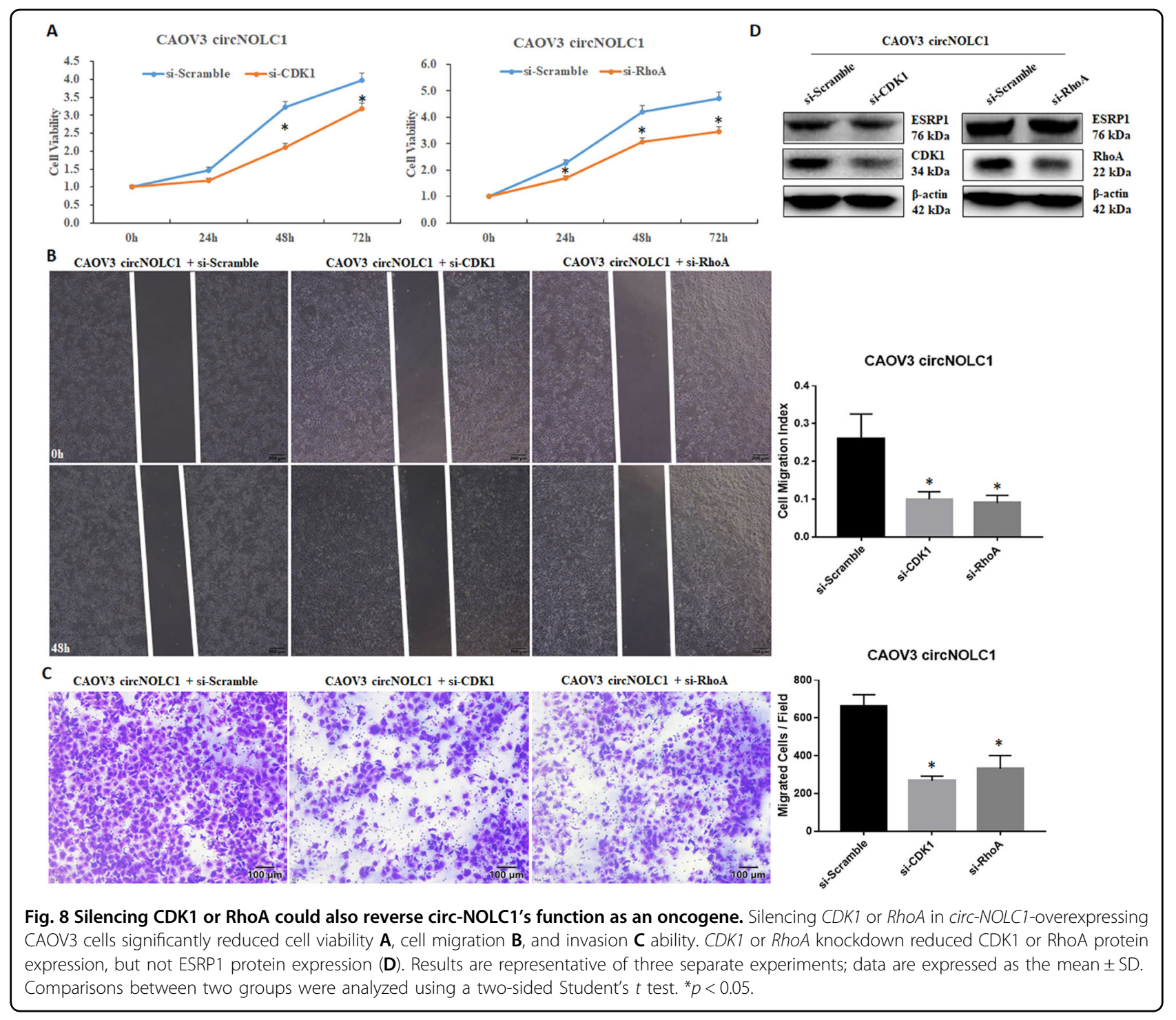

placed in an incubator for $3-4 \mathrm{~h}$ for coagulation. Then, $200 \mu \mathrm{L}$ of serum-free medium containing 50,000 resuspended cells was added to the upper chamber, $600 \mu \mathrm{L}$ of complete cell culture medium was added as a chemoattractant to the lower chamber, and after incubation for $48 \mathrm{~h}$, the chambers were washed with PBS three times, fixed with $5 \%$ paraformaldehyde for $15 \mathrm{~min}$, and then stained with a crystal violet solution for $15 \mathrm{~min}$. Finally, the cells were counted under a microscope (Olympus, Tokyo, Japan) to evaluate cell invasion.

\section{Plate clone formation assay}

Cells (200 cells per well) were seeded into a six-well plate, and then incubated in $5 \% \mathrm{CO}_{2}$ and $37^{\circ} \mathrm{C}$ for 2 weeks (complete medium) until macroscopic cell clones appeared. The cells were carefully washed using PBS, fixed using $4 \%$ paraformaldehyde for $15 \mathrm{~min}$, stained with
Giemsa solution for $15 \mathrm{~min}$, washed with tap water, and air-dried. The colony formation rate was calculated using the following formula: (number of clones/number of cells inoculated) $\times 100 \%$.

\section{Apoptosis assays}

Cells were collected and resuspended with $100 \mu \mathrm{L} 1 \times$ buffer containing phycoerythrin-labeled annexin $\mathrm{V}$ and 7aminoactinomycin $\mathrm{D}(5 \mu \mathrm{L}$ each, BD) in the dark for $15 \mathrm{~min}$. Then, $400 \mu \mathrm{L}$ of buffer was added and the rate of cell apoptosis was determined using flow cytometry within one hour.

\section{QRT-PCR}

Total RNA of tissue and cell samples was extracted using the TRIzol reagent (Takara, Shiga, Japan). Chloroform $(200 \mu \mathrm{L})$ was added into $1 \mathrm{~mL}$ of TRIzol, and after 
centrifugation, the upper aqueous phase was aspirated into a new tube and an equal volume of isopropanol was added to precipitate the RNA. After centrifugation, the supernatant was discarded and $75 \%$ ethanol was used to wash the RNA pellet. The precipitate was dried and dissolved in diethylpyrocarbonate water. The OD value at $260 \mathrm{~nm}$ was measured using an ultraviolet spectrophotometer (Unico, Shanghai, China) to calculate the concentration of RNA. Then, RNA was reversetranscribed to cDNA according to the manufacturer's instructions (Takara). Next, real-time quantitative PCR amplification of cDNA was carried out using a SYBR PrimeX EX-TAQ Patent II Kit (Takara, Shiga, Japan). Finally, the cycle threshold $(\mathrm{Ct})$ of the target gene and $18 \mathrm{~S}$ rRNA (18 s) were compared using the $2-\Delta \Delta \mathrm{Ct}$ method (GenePharma) to determine relative expression levels of the target gene.

\section{Western blotting}

Cells were lysed with radioimmunoprecipitation assay buffer containing protease inhibitors. After quantification, $40 \mu \mathrm{g}$ of denatured protein was separated using 10\% sodium dodecyl sulfate-polyacrylamide gel electrophoresis, followed by electro-transfer onto a polyvinylidene difluoride membrane that had already been pre-activated in methanol. The membrane was then washed with trisbuffered saline and Tween 20 (TBST) for 1-2 min, blocked with $3 \%$ bovine serum albumin at room temperature for $1-2 \mathrm{~h}$. Thereafter, the membranes were incubated with primary antibodies against ESRP1 (Cat No.: 21045-1-AP), ras homolog family member A (RhoA, Cat No.: 10749-1-AP), CDK1 (Cat No.: 19532-1-AP) (1:1000, Proteintech Group, Chicago, IL, USA) and $\beta$-actin (1:3000, Cat No.: 20536-1-AP, Proteintech Group) overnight at $4{ }^{\circ} \mathrm{C}$. On the 2 nd day, the membrane was washed with TBST three times, and then incubated with anti-rabbit secondary antibodies at room temperature for $2 \mathrm{~h}$. After washing with TBST again three times, the immunoreactive proteins were visualized using the ECL system (Santa Cruz Biotechnology, Santa Cruz, CA, USA).

\section{RNA-binding protein immunoprecipitation (RIP) assay}

Bioinformatic prediction (catRAPID, http://s. tartaglialab.com/page/catrapid_group) revealed that ESRP1 protein could bind with circ-NOLC1. The Magna RIP RNA-Binding Protein Immunoprecipitation Kit (Millipore, Bedford, MA, USA) was used for the RIP assay. In brief, A2780 cells were lysed in RIP lysis buffer and the cell extract was incubated with RIP buffer containing magnetic beads conjugated to human anti-ESRP1 antibodies or normal rabbit IgG as a negative control. Thereafter, to digest the proteins, the samples were incubated with proteinase $\mathrm{K}$. The immunoprecipitated RNA was isolated and determined using qRT-PCR analysis. Control amplification was carried out on input RNA before immunoprecipitation, which was set as the positive control.

\section{Subcutaneous tumor dissemination assay}

BALB/c nude mice (Vital River Laboratories, Beijing, China) were raised in a specific pathogen-free environment. In brief, after serpentine grouping by weight, $1 \times 10^{7}$ circ-NOLC1-downregulated A2780 cells or control A2780 cells in $150 \mu \mathrm{L}$ FBS-free media were injected into 5-week-old female mice subcutaneously to establish the subcutaneous dissemination model. At appropriate times after injection (Tumor size $<2 \mathrm{~cm}$ ), the mice were euthanized and the tumor nodes were resected and measured. All animal experiments were approved by China Medical University Animal Care and Use Committee and were carried out following the Guide for the Care and Use of Laboratory Animals (published by the National Institute of Health). No blinding was done.

\section{Dual-luciferase reporter assay}

The wild-type or mutated PSI-check2 Dual-luciferase vectors containing miR-326-5p, miR-330, miR-370, and miR-9-5p binding sites on circ-NOLC1 (Hanbio Biotechnology, Shanghai, China) were co-transfected with miR-326-5p, miR-330, miR-370, and miR-9-5p mimics or scrambled controls into the HEK-293T cells. Cell extracts were prepared to measure the luciferase activity using the Dual-Luciferase Reporter Assay System (Promega, Madison, WI, USA). The relative luciferase signal was determined by the normalization of firefly luciferase activity to that of Renilla luciferase.

\section{Statistical analysis}

Statistical analyses were performed using SPSS 20.0 software (IBM Corp., Armonk, NY, USA). Experiments were repeated three times, and the mean \pm SD was used in each group of data to reflect the overall parameters. Comparisons between two groups were analyzed using a two-sided Student's $t$ test. Correlation between circ-NOLC1 expression and clinicopathological characteristics of the patients with ovarian cancer were examined using the $X^{2}$ test. $P<0.05$ was considered statistically significant.

\section{Acknowledgements}

This study was supported by the National Natural Science Foundation of China (nos. 81872115; 81772776; 81772785) and the Young Science-technology Talents Support Project of Guangzhou (no. X20200301068).

\footnotetext{
Author details

${ }^{1}$ Department of Gynecologic Oncology Research Office, The Third Affiliated Hospital of Guangzhou Medical University, Guangzhou 510150, China.

Department of Obstetrics and Gynecology, Center for Reproductive Medicine/ Department of Fetal Medicine and Prenatal Diagnosis, Key Laboratory for Major
} 
Obstetric Diseases of Guangdong Province, The Third Affiliated Hospital of Guangzhou Medical University, Guangzhou 510150, China. ${ }^{3}$ Department of Gynecology, The First Affiliated Hospital of China Medical University, Shenyang 110001, China. ${ }^{4}$ Oral and Biomedical Sciences, School of Dentistry, Cardiff University, CF14 4XY Cardiff, UK

\section{Conflict of interest}

The authors declare that they have no conflict of interest.

\section{Publisher's note}

Springer Nature remains neutral with regard to jurisdictional claims in published maps and institutional affiliations.

The online version of this article (https://doi.org/10.1038/s41420-020-00381-0) contains supplementary material, which is available to authorized users.

Received: 20 May 2020 Revised: 19 November 2020 Accepted: 24 November 2020

Published online: 22 January 2021

\section{References}

1. Torre, L. A. et al. Ovarian cancer statistics, 2018. CA Cancer J. Clin. 68, 284-296 (2018).

2. Armstrong, D. K. et al. NCCN Guidelines Insights: ovarian cancer, version 1.2019. J. Natl. Compr. Canc. Netw. 17, 896-909 (2019).

3. O'Connor, K. L. \& Mercurio, A. M. Protein kinase A regulates Rac and is required for the growth factor-stimulated migration of carcinoma cells. J. Biol. Chem. 276, 47895-47900 (2001).

4. Menon, U., Gentry-Maharaj, A. \& Jacobs, I. Ovarian cancer screening and mortality. JAMA 306, 1544 (2011).

5. Haruta, S. et al. Molecular genetics and epidemiology of epithelial ovarian cancer. Oncol. Rep. 26, 1347-56 (2011).

6. Chen, S. \& Zhao, Y. Circular RNAs: characteristics, function, and role in human cancer. Histol. Histopathol. 33, 887-893 (2018).

7. Chen, L. L. \& Yang, L. Regulation of circRNA biogenesis. RNA Biol. 12, 381-8 (2015).

8. Salzman, J., Chen, R. E., Olsen, M. N., Wang, P. L. \& Brown, P. O. Cell-type specific features of circular RNA expression. PLoS Genet. 9, e1003777 (2013).

9. Zhang, X. O. et al. Diverse alternative back-splicing and alternative splicing landscape of circular RNAs. Genome Res. 26, 1277-87 (2016).

10. Kulcheski, F. R., Christoff, A. P. \& Margis, R. Circular RNAs are miRNA sponges and can be used as a new class of biomarker. J. Biotechnol. 238, 42-51 (2016).

11. Zhong, Z., LV, M. \& Chen, J. Screening differential circular RNA expression profiles reveals the regulatory role of circTCF25-miR-103a-3p/miR-107-CDK6 pathway in bladder carcinoma. Sci. Rep. 6, 30919 (2016).

12. Luo, Y. et al. CircRNA_101505 sensitizes hepatocellular carcinoma cells to cisplatin by sponging miR-103 and promotes oxidored-nitro domain-containing protein 1 expression. Cell Death Discov. 5, 121 (2019).

13. Yang, Y. et al. Extensive translation of circular RNAs driven by N6methyladenosine. Cell Res. 27, 626-641 (2017).
14. Li, Z. et al. Exon-intron circular RNAs regulate transcription in the nucleus. Nat. Struct. Mol. Biol. 22, 256-64 (2015).

15. Liu, Z. et al. Circular RNA clARS regulates ferroptosis in HCC cells through interacting with RNA binding protein ALKBH5. Cell Death Discov. 6, 72 (2020).

16. Du, W. W. et al. Foxo3 circular RNA retards cell cycle progression via forming ternary complexes with p21 and CDK2. Nucleic Acids Res. 44, 2846-58 (2016).

17. Tsai, Y. T. et al. Chromatin tethering effects of hNopp140 are involved in the spatial organization of nucleolus and the rRNA gene transcription. J. Biomed. Sci. 15, 471-486 (2008).

18. Thiry, M. et al. Localization of Nopp140 within mammalian cells during interphase and mitosis. Histochem. Cell Biol. 132, 129-140 (2009).

19. Zhu, C. et al. The interaction between NOLC1 and IAV NS1 protein promotes host cell apoptosis and reduces virus replication. Oncotarget 8, 94519-94527 (2017).

20. Wilusz, J. E. \& Sharp, P. A. Molecular biology. A circuitous route to noncoding RNA. Science 340, 440-441 (2013).

21. Hall, A. Rho GTPases and the actin cytoskeleton. Science 279, 509-514 (1998).

22. DerMardirossian, C. \& Bokoch, G. M. GDIs: central regulatory molecules in Rho GTPase activation. Trends Cell Biol. 15, 356-363 (2005).

23. Liu, J. et al. PI3K/Akt-dependent phosphorylation of GSK3 3 and activation of RhoA regulate Wnt5a-induced gastric cancer cell migration. Cell Signal. 25, 447-456 (2013).

24. Olofsson, B. Rho guanine dissociation inhibitors: pivotal molecules in cellular signalling. Cell Signal 11, 545-554 (1999).

25. Chen, S. et al. The involvement of RhoA and Wnt-5a in the tumorigenesis and progression of ovarian epithelial carcinoma. Int J. Mol. Sci. 14, 24187-24199 (2013).

26. Nigg, E. A. Mitotic kinases as regulators of cell division and its checkpoints. Nat. Rev. Mol. Cell Biol. 2, 21-32 (2001).

27. Chen, S., Chen, X., Xiu, Y. L., Sun, K. X. \& Zhao, Y. MicroRNA-490-3P targets CDK1 and inhibits ovarian epithelial carcinoma tumorigenesis and progression. Cancer Lett. 362, 122-30 (2015).

28. Hentze, M. W. \& Preiss, T. Circular rnas: Splicing's enigma variations. EMBO J. 32 923-925 (2013).

29. Abdelmohsen, K. et al. Identification of hur target circular mas uncovers suppression of pabpn1 translation by circpabpn1. RNA Biol. 14, 361-369 (2017).

30. Wang, L. L. et al. CircRhoC promotes tumorigenicity and progression in ovarian cancer by functioning as a miR-302e sponge to positively regulate VEGFA. J. Cell Mol. Med 23, 8472-8481 (2019).

31. Sun, Y. M. et al. CircMYBL2, a circRNA from MYBL2, Regulates FLT3 Translation by Recruiting PTBP1 to Promote FLT3-TTD AML Progression. Blood 134, 1533-1546 (2019)

32. Warzecha, C. C., Sato, T. K., Nabet, B., Hogenesch, J. B. \& Carstens, R. P. ESRP1 and ESRP2 are epithelial cell-type-specific regulators of FGFR2 splicing. Mol. Cell 33, 591-601 (2009).

33. Yae, T. et al. Alternative splicing of CD44 mRNA by ESRP1 enhances lung colonization of metastatic cancer cell. Nat. Commun. 3, 883 (2012).

34. Fagoonee, S. et al. The RNA-binding protein ESRP1 promotes human colorectal cancer progression. Oncotarget 8, 10007-10024 (2017).

35. Jeong, H. M. et al. ESRP1 is overexpressed in ovarian cancer and promotes switching from mesenchymal to epithelial phenotype in ovarian cancer cells. Oncogenesis 6, e391 (2017). 